\section{Lung cancer screening: improving understanding of the psychological impact}

\author{
Samantha L Quaife, ${ }^{1}$ Sam M Janes ${ }^{2}$
}

The clinical evidence base for low-dose CT (LDCT) lung cancer screening is rapidly advancing and the case for implementing a UK programme building. This is welcome news to the lung cancer community whose patients too often face a bleak prognosis, in part because early diagnosis is so infrequent. Relatively less attention, however, has been paid to the potential psychological responses of high-risk individuals to such a screening programme; something which should inform policy decision-makers and shape healthcare professionals' practice of screening, to maximise patient benefit and well-being.

Previous research on screening for other types of cancer has shown there is potential for patients to experience distress at any point along the screening pathway. An individual's propensity to experience distress in response to cancer screening may be determined in part by the nature of the result they receive and any ensuing tests or surveillance, as well as their psychosocial characteristics. ${ }^{1}$ In Thorax, Brain et al provide an invaluable contribution to both these aspects and the first from the UK; reporting the longterm psychosocial outcomes of screening among participants in the UK Lung Screening (UKLS) trial. $^{2}$

The psychological cost of abnormal findings is especially important to understand in the context of LDCT lung cancer screening because they are frequent, with an average pulmonary nodule detection rate of $20 \%$ across trials. ${ }^{3}$ In the US National Lung Screening Trial (NLST), $39 \%$ of participants received a positive screening result for one of their three annual screens, of which $96 \%$ were false positives. ${ }^{4}$ Incidental findings (ie, medical conditions other than lung cancer) were also a common outcome for screenees,

\footnotetext{
${ }^{1}$ Health Behaviour Research Centre, Department of Epidemiology and Public Health, University College London, London, UK; ' 2 Lungs for Living Research Centre, UCL Respiratory, Division of Medicine, Rayne Building, University College London, London, UK

Correspondence to Professor Sam M Janes, Lungs for Living Research Centre, UCL Respiratory, Division of Medicine, Rayne Building, University College London, London WC1E 6JF, UK;

s.janes@ucl.ac.uk
}

affecting $10 \%$ of NLST participants following their prevalence (first) screen. ${ }^{4}$ The UKLS investigators made a distinction between false positive and indeterminate results based on their clinical management. Pulmonary nodules which were immediately suspicious for cancer and referred to a multidisciplinary team were categorised as 'false positive' if found to be benign, whereas nodules under surveillance using interval scans were termed 'indeterminate'. The rates were $3.6 \%$ and $23 \%$, respectively, similar to the Dutch-Belgian trial NELSON. $^{5}$

A diagnosis of lung cancer carries significant distress, but any abnormality associated with lung cancer could induce anxiety, and uncertain or prolonged periods of surveillance may be difficult to tolerate. Brain et al found that distress was significantly increased among UKLS participants receiving abnormal screening results and requiring repeat imaging. However, these effects were diminished at the 2 years' follow-up and did not breach clinically significant thresholds. Anxiety was highest among those under investigation for major lung abnormalities, but again this anxiety appeared to be resolved at follow-up. Previous trials have also observed significant short-term screening-induced distress, ${ }^{6-9}$ and similarly, systematic reviews of this area have found no evidence that this distress persists beyond 6 months. ${ }^{10} 11$ Among NLST participants, those diagnosed with lung cancer reported impairments in health-related quality of life but this was not true for other abnormal results at either 1-month or 6-month follow-up, relative to those receiving negative results. ${ }^{12}$

The UKLS findings are therefore promising, as are those from other screening trials. However, as the authors acknowledge, longer term adverse psychological outcomes should continue to be researched because there are important differences between the trial and health service contexts. The most obvious is that the UKLS trial participants were a self-selected group, and there is evidence from the Danish Lung Cancer Screening Trial (DLCST) to suggest they could be less vulnerable to distress. ${ }^{13}$ Indeed, disparities in participation have been a particular problem for lung cancer screening. Fewer current smokers and individuals of a lower socioeconomic position have participated across European and US trials; ${ }^{13}{ }^{14}$ a paradox whereby those at higher risk and most likely to benefit appear to be less likely to consider screening. Importantly, Brain et al also identified current smoking status as a potential risk factor for heightened screening distress. While socioeconomic deprivation was not independently associated with distress, the Liverpool recruitment site was, where levels of deprivation were highest. Distress was also higher among women (again, fewer of whom took part in UKLS), those who were younger and individuals who had experience of lung cancer. Poorer health-related quality of life and increased anxiety have previously been observed among current smokers, those with a longer smoking history, and individuals with a lower level of education in the $\mathrm{NELSON}^{6}{ }^{7}$ and $\mathrm{PLuSS}^{8}$ screening cohorts.

Therefore, the UKLS results suggest that some of the same factors associated with non-participation and increased risk of lung cancer may constitute risk factors for worse psychosocial outcomes. From a psychological perspective, this touches on an important deviation of lung cancer screening from existing UK cancer screening programmes: the invitation of those at high risk and predominantly due to a behavioural risk factor. Baseline levels of lung cancer worry and distress could therefore already be high, to which screening has the potential to contribute and also relieve and reassure. For some, screening may prompt conscious awareness of risk and a prospect of diagnosis that is difficult to cope with, potentially exacerbated by the perceived stigmatisation of smoking-related risk. Higher affective perceptions of risk $^{15}$ and guilt about smoking ${ }^{16}$ have been shown to predict higher screening-related distress. Indeed, there is some evidence (although mixed) to suggest that enrolment into a lung cancer screening trial causes distress, with the DLCST trial finding higher levels of distress among the no-screen controls. ${ }^{17}$ This is contrary to the results of the UKLS study, and probably explained by the fact that the DLCST control group were also required to regularly attend clinics. This would not be an issue in the event of a national screening programme because all those who are at high risk and eligible would subsequently be offered the screening test. Nevertheless, it is interesting because it implies that screening may offer a means of managing anxiety about higher perceived risk. Smokers have been shown to have more fatalistic views 
towards cancer ${ }^{18}$ and perhaps screening could provide a means of instilling a greater sense of control over respiratory health and risk of lung cancer mortality that long-term addiction to tobacco may have quashed. Indeed, concern has been raised about the interpretation of a negative screen as a 'licence to smoke', but alternatively positive responses could be researched and capitalised on to promote smoking cessation. Studies are needed which examine lung cancer-related distress against a comparable high-risk sample who are blind to the prospect of screening.

If the opportunity arises in the UK, the full spectrum of psychological responses to a regular long-term screening programme needs also to be measured. The accumulation of abnormal findings, or the reassurance offered by long-term enrolment in a regular programme, could lead to different outcomes. Potential implications of abnormal results for screening engagement should also be examined as the UKLS study found lower decisional satisfaction (about taking part in the trial) among participants recalled for a repeat scan, and in the Prostate, Lung, Colorectal and Ovarian cancer screening trial, false positive results predicted poorer trial adherence. ${ }^{19}$ The development and use of condition-specific measures, such as that by Brodersen et al, ${ }^{20}$ may aid such a research agenda. Specific measures are likely to be more sensitive, and have the potential to advance understanding of the root causes of distress among specific subgroups from which targeted interventions could be developed. More generally, and to share the conclusions of Brain et al, supportive interventions and communication strategies, especially for those identified as at greater risk of distress, should be developed in adjunct to the implementation of screening in the event of a national programme. Previous studies of pulmonary nodules detected incidentally find that the quality of communication is positively associated with psychological well-being. ${ }^{21}$ The same care and attention paid by trials to prescreening counselling should be translated to a screening service. Information and communication strategies will need to be developed that prepare individuals psychologically for abnormal screening results so that they are fully informed.

The UKLS study is therefore a hugely important and timely contribution, which has greatly advanced our understanding of the psychosocial impact of lung cancer screening and those subgroups who may be more vulnerable to experiencing distress. It provides the foundations for future research to investigate risk factors for increased distress, to examine the full spectrum of psychological responses to lung cancer screening in the health service context, and to develop supportive communication strategies and interventions that further increase the benefits of screening.

Correction notice This article has been corrected since it was published Online First. The authors affiliation and author correspondence information has been updated.

Funding SLQ is supported by the Medical Research Council. SMJ is a Wellcome Trust Senior Fellow in Clinical Science and is supported by the Roy Castle Lung Cancer Foundation and CRUK Lung Cancer Centre of Excellence and UCLH/UCL BRC.

Competing interests None declared.

Provenance and peer review Commissioned; externally peer reviewed.

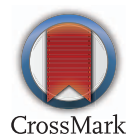

To cite Quaife SL, Janes SM. Thorax 2016;71:971972.

Published Online First 31 August 2016

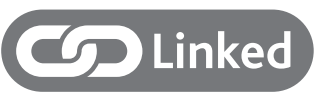

http://dx.doi.org/10.1136/thoraxjnl-2016-208283

Thorax 2016;71:971-972.

doi:10.1136/thoraxjnl-2016-208966

\section{REFERENCES}

1 Wardle J, Pope R. The psychological costs of screening for cancer. J Psychosom Res 1992;36:609-24

2 Brain K, Lifford KJ, Carter B, et al. Long-term psychosocial outcomes of low-dose CT screening: results of the UK Lung Cancer Screening randomised controlled trial. Thorax 2016;71:996-1005.

3 Bach PB, Mirkin JN, Oliver TK, et al. Benefits and harms of CT screening for lung cancer: a systematic review. JAMA 2012;307:2418-29.

4 National Lung Screening Trial Research Team, Aberle DR, Adams AM, et al. Reduced lung-cancer mortality with low-dose computed tomographic screening. N Engl J Med 2011;365:395-409.

5 Field JK, Duffy SW, Baldwin DR, et al. UK Lung Cancer RCT Pilot Screening Trial: baseline findings from the screening arm provide evidence for the potential implementation of lung cancer screening. Thorax 2016;71:161-70.

6 van den Bergh KAM, Essink-Bot ML, Borsboom GJJM, et al. Short-term health-related quality of life consequences in a lung cancer $\mathrm{CT}$ screening trial (NELSON). Br J Cancer 2010;102:27-34.

7 van den Bergh KAM, Essink-Bot ML, Borsboom GJJM, et al. Long-term effects of lung cancer computed tomography screening on health-related quality of life: the NELSON trial. Eur Respir J 2011;38:154-61.

8 Byrne MM, Weissfeld J, Roberts MS. Anxiety, fear of cancer, and perceived risk of cancer following lung cancer screening. Med Decis Making 2008;28:917-25.

9 Taylor KL, Shelby R, Gelmann E, et al. Quality of life and trial adherence among participants in the prostate, lung, colorectal, and ovarian cancer screening trial. J Natl Cancer Inst 2004;96: 1083-94.

10 Slatore CG, Sullivan DR, Pappas M, et al. Patient-centered outcomes among lung cancer screening recipients with computed tomography: a systematic review. I Thorac Oncol 2014;9: 927-34.

11 Wu GX, Raz DJ, Brown L, et al. Psychological burden associated with lung cancer screening: a systematic review. Clin Lung Cancer. Published Online: 30 Mar 2016. doi: 10.1016/j.cllc.2016.03.007

12 Gareen IF, Duan F, Greco EM, et al. Impact of lung cancer screening results on participant health-related quality of life and state anxiety in the National Lung Screening Trial. Cancer 2014;120:3401-9.

13 Hestbech MS, Siersma V, Dirksen A, et al. Participation bias in a randomised trial of screening for lung cancer. Lung Cancer 2011;73:325-31.

14 National Lung Screening Trial Research Team, Aberle DR, Adams AM, et al. Baseline characteristics of participants in the randomized national lung screening trial. J Nat/ Cancer Inst 2010;102: 1771-9.

15 Bunge EM, van den Bergh KAM, Essink-Bot ML, et al. High affective risk perception is associated with more lung cancer-specific distress in $\mathrm{CT}$ screening for lung cancer. Lung Cancer 2008:62:385-90.

16 Plank A, Nemesure $B$, Bilfinger $T$, et al. Lung cancer screening and self-reported distress. Chest 2014;146:594A.

17 Aggestrup LM, Hestbech MS, Siersma V, et al. Psychosocial consequences of allocation to lung cancer screening: a randomised controlled trial. BMJ Open 2012;2:e000663.

18 Quaife SL, McEwen A, Janes SM, et al. Smoking is associated with pessimistic and avoidant beliefs about cancer: results from the International Cancer Benchmarking Partnership. $\mathrm{Br}$ I Cancer 2015;112:1799-804.

19 Ford ME, Havstad SL, Flickinger L, et al. Examining the effects of false positive lung cancer screening results on subsequent lung cancer screening adherence. Cancer Epidemiol Biomarkers Prev 2003;12:28-33.

20 Brodersen J, Thorsen $\mathrm{H}$, Kreiner $\mathrm{S}$. Consequences of screening in lung cancer: development and dimensionality of a questionnaire. Value Health 2010;13:601-12.

21 Slatore CG, Golden SE, Ganzini L, et al. Distress and patient-centered communication among veterans with incidental (not screen-detected) pulmonary nodules. A cohort study. Ann Am Thorac Soc 2015;12:184-92. 